\title{
Noncanonical Wnt5a Signaling Suppresses Hippol TAZ-Mediated Osteogenesis Partly Through the Canonical Wnt Pathway in SCAPs
}

\author{
Yajing Fu (D)', Dan Ma², Fengyan Fan², Tongke Sun', Ruiqi Han', Yanran Yang', Jun Zhang' \\ 'Shandong University \& Shandong Key Laboratory of Oral Tissue Regeneration \& Shandong Engineering Laboratory for Dental Materials and Oral \\ Tissue Regeneration, Department of Orthodontics, School and Hospital of Stomatology, Cheeloo College of Medicine, Shandong University, Jinan, \\ People's Republic of China; ${ }^{2}$ Department of Orthodontics, Shandong Provincial Hospital Affiliated to Shandong First Medical University, Jinan, People's \\ Republic of China; ${ }^{3}$ Department of Orthodontics, Hangzhou Stomatological Hospital, Hangzhou, People's Republic of China \\ Correspondence: Jun Zhang, Shandong University \& Shandong Key Laboratory of Oral Tissue Regeneration \& Shandong Engineering Laboratory for \\ Dental Materials and Oral Tissue Regeneration, Department of Orthodontics, School and Hospital of Stomatology, Cheeloo College of Medicine, \\ Shandong University, No. 44-I Wenhua Road West, Jinan, People’s Republic of China, Tel +86 I39 5310 98I6, Email zhangj@sdu.edu.cn
}

Purpose: Stem cells from the apical papilla (SCAPs) are promising seed cells for tissue regeneration medicine and possess the osteogenic differentiation potential. Wnt5a, a typical ligand of the noncanonical Wnt pathway, exhibits diverse roles in the regulation of osteogenesis. The transcriptional co-activator with PDZ-binding motif (TAZ, WWTR1) is a core regulator in the Hippo pathway and regulates stem behavior including osteogenic differentiation. This study aims to examine how Wnt5a regulates SCAPs osteogenesis and explore the precise mechanistic relationship between Wnt5a and TAZ.

Methods: SCAPs were isolated from developing apical papilla tissue of extracted human immature third molars in vitro. ALP staining, ALP activity and Alizarin red staining were used to evaluate osteogenic capacity. Osteogenic-related factors were assessed by qRT-PCR or Western blotting. Additionally, the receptor tyrosine kinase-like orphan receptor 2 (ROR2) was detected by immunocytofluorescence staining and silenced by small interfering RNA to verify the function of Wnt5a/ROR2 in TAZ-mediated osteogenesis. And we constructed TAZ-overexpression and $\beta$-catenin-overexpression SCAPs generated by lentivirus to explore the precise mechanistic relationship between Wnt5a and TAZ.

Results: Wnt5a (100ng/mL) significantly suppressed ALP activity, mineralization nodules formation, expression of osteogenic-related factors. Meanwhile, it decreased the expression of TAZ mRNA and protein. TAZ overexpression promoted osteogenesis of SCAPs while Wnt5a could block TAZ-mediated osteogenesis. Furthermore, ROR2 siRNA (siROR2) was found to upregulate TAZ and canonical Wnt pathway signaling related molecules such as $\beta$-catenin, GSK3 $\beta$ and p-GSK3 $\beta$. The suppression of Wnt5a/ROR2 on osteogenesis was significantly reversed by $\beta$-catenin overexpression through Wnt5a/ROR2/ $\beta$-catenin/TAZ pathway.

Conclusion: Taken together, the present study demonstrates that Wnt5a suppresses TAZ-mediated osteogenesis of SCAPs and there may be a Wnt5a/ROR2/ $\beta$-catenin/TAZ pathway regulating osteogenesis of SCAPs. Moreover, Wnt5a could be a candidate for regulators in tissue regeneration.

Keywords: Wnt5a, TAZ, stem cells from the apical papilla, osteogenesis, $\beta$-catenin

\section{Introduction}

With the development of stem cell technology, stem cell-based tissue regeneration therapy is rapidly moving into clinical application. Recently, dental-tissue-derived MSCs have been extensively studied concerning their characteristics of selfrenewal, proliferation, and differentiation. Stem cells from the apical papilla (SCAPs) are multipotent stem cells retained at the root of immature permanent teeth. A multitude of evidence describes that SCAPs have the capacity for differentiation into chondrogenic, osteogenic, and neurogenic tissue. ${ }^{1-3}$ Besides, there is a trend that alveolar bone tends to degenerate, which results in appearance of impacted third molar teeth. And therefore, extraction of impacted and 


\section{Graphical Abstarct}

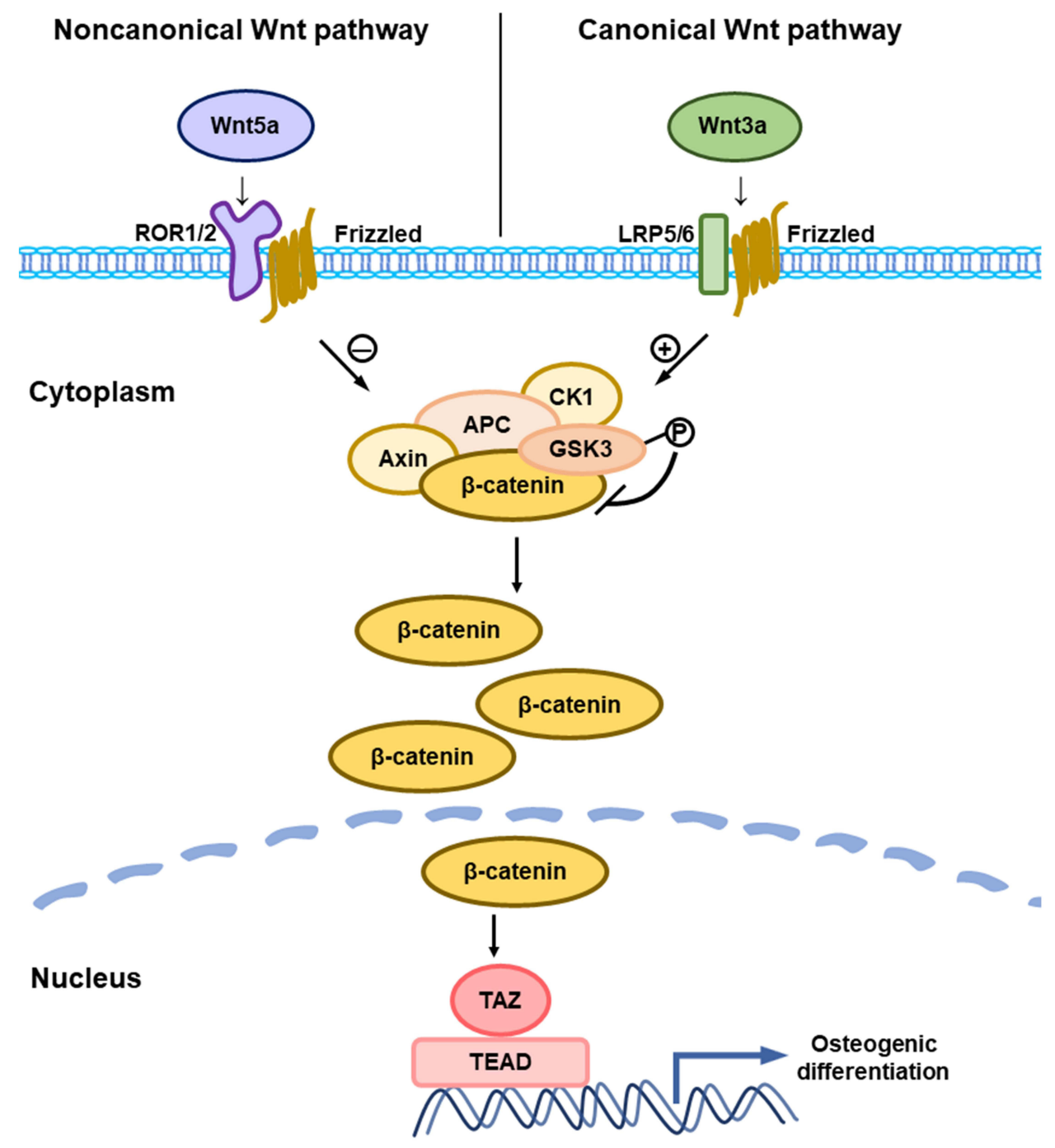

immature third molars offers abundant SCAPs for stem cell-based tissue regeneration therapy. Because of these characteristics, SCAPs are promising seed cells for tissue regeneration medicine.

The Hippo pathway is highly conserved and plays an essential role in organ development, tissue homeostasis, and neoplasia. The transcriptional co-activator with PDZ-binding motif (TAZ, WWTR1) is one of core regulators among Hippo pathway signaling molecules, reportedly regulating stem cell behavior and regeneration via a post-translational mechanism. ${ }^{4,5}$ To date, evidence suggests that TAZ-mediated osteogenesis can be referred to in many cellular physiologies such as mechanotransduction and BMP-2 signaling. ${ }^{6-9}$ Besides, TAZ, located in the dental papilla and the entire enamel organ, mediates signaling center formation, the enamel knot during tooth development. ${ }^{10,11}$ Thus, Hippo/TAZ may be important crucial for regulating osteogenic differentiation in SCAPs.

Wnts are a family consisting of 19 secreted glycoproteins that are extensively involved in fundamental bone metabolism such as osteoblast proliferation, differentiation, and apoptosis. ${ }^{12-14}$ Wnt $5 \mathrm{a}$ is a representative ligand of noncanonical Wnt pathway and exhibit potentiality on stemness maintenance and osteogenesis of MSCs ex vivo. ${ }^{15}$ Mice deficient in Wnt5a exhibited a low bone mass with decreased bone formation, ${ }^{14}$ retarded tooth development and dis- 
regulated cusp patterning. ${ }^{16}$ Additionally, Wnt5a/ROR2 signaling involves in BMP-2-mediated osteoblast differentiation in a Smad-independent pathway. ${ }^{17}$ It also enhances receptor activation of nuclear factor- $\kappa \mathrm{B}$ (RANK) expression in osteoclast precursors by activating JNK and recruiting c-Jun on the promoter of the gene encoding RANK, thereby enhancing RANK ligand (RANKL)-induced osteoclastogenesis. ${ }^{18}$ However, whether Wnt5a affects osteogenic differentiation of SCAPs has not yet been described and the detailed mechanism remains unclear.

Recently, cross talk between the Wnt and Hippo signaling pathways has been investigated to uncover the precise regulatory mechanisms involved. Azzolin and others found that the main regulator of the canonical Wnt pathway, APC, is responsible for TAZ degradation in the cytoplasm, and the key effector, $\beta$-catenin, is required for the interaction of TAZ with $\beta$-TrCP. ${ }^{19,20}$ Moreover, research has verified that Wnt3a induces nuclear localisation of TAZ during osteogenic differentiation. ${ }^{21}$ However, a relationship between the noncanonical Wnt pathway and the the Hippo pathway is rarely reported. Hyun WooPark and others have even referred to a Wnt5a/b-YAP/TAZ signaling axis, that is, Wnt-FZD/RORGa12/13-Rho-Lats1/2-YAP/TAZ. They have identified its involvement in osteogenesis and cell migration. ${ }^{22}$ Other research focused on kidney fibrosis indicated that Wnt5a stimulates Yap/Taz-mediated macrophage M2 polarization. ${ }^{23}$ Based on previous studies, our team put forward a hypothesis that Wnt5a may regulate the osteogenesis of SCAPs via the Hippo/TAZ pathway. This study aims to examine how Wnt5a regulates SCAP osteogenesis and explore the precise mechanistic relationship between Wnt5a and TAZ.

\section{Materials and Methods}

\section{Isolation and Cultivation of SCAPs}

SCAPs were isolated from the developing apical papilla tissue of extracted human immature third molars. The inclusion criteria were as follows: indication for the extracted of third molar teeth; radiographic evidence of an immature stage of development with open apices $(>1.5 \mathrm{~mm})$; patients between 13-25 years of age; willingness to participate in the study; ability of the patient (or parent/guardian when the patient was $<18$ years of age) to understand and provide informed consent. The exclusion criterion was no other indication for the extraction of teeth (eg, carious lesions or pulpal inflammation/necrosis). ${ }^{24,25}$ All patients (or parent/guardian when the patient was $<18$ years of age) gave their informed consent for inclusion before they participated in the study. The study was conducted in accordance with the Declaration of Helsinki, and the protocol (No. R20180701) was approved and supervised by the Ethics Committee of the Hospital of Stomatology, Shandong University (Jinan, China). The isolated tissue was digested with $3 \mathrm{mg} / \mathrm{mL}$ collagenase type I (Solarbio, Beijing, China) and $4 \mathrm{mg} / \mathrm{mL}$ dispase II (Sigma, Darmstadt, Germany) at $37{ }^{\circ} \mathrm{C}$ for an hour. SCAPs were grown in minimum essential medium Eagle alpha modification ( $\alpha \mathrm{MEM}$; BI, Kibbutz Beit-Haemek, Israel) supplemented with 10\% fetal bovine serum (FBS) (BI, Kibbutz Beit-Haemek, Israel), $100 \mathrm{U} / \mathrm{mL}$ penicillin, and $100 \mu \mathrm{g} / \mathrm{mL}$ streptomycin (Biosharp, Anhui, China). Cells were incubated at 5\% CO2 and $37^{\circ} \mathrm{C}$.

\section{Colony Formation Assay}

A total of 200 SCAPs were plated on a 6 -cm diameter culture dish for 10 days in $10 \%$ FBS/ $\alpha$-MEM. The cells were fixed with 4\% paraformaldehyde (PFA), washed with phosphate buffer saline (PBS), and stained with crystal violet (Solarbio). The cells were then washed and dried. Cell clusters of more than 50 cells were counted as one colony. The colony counting was captured under a microscope (Olympus, Japan).

\section{Alkaline Phosphatase (ALP) Activity and ALP Staining Assay}

SCAPs were plated in 6-well plates and cultured in osteogenic inducing medium with or without Wnt5a (R\&D, Minnesota, USA). ALP activity was quantified using an alkaline phosphatase assay kit (Nanjing Jiancheng Bioengineering Institute, Nanjing, China). Cell culture medium were collected after 3 or 7 days stimuli. The collected solution were centrifuged at $1000 \mathrm{rpm}$ for $5 \mathrm{~min}$ at $4{ }^{\circ} \mathrm{C}$. Then, an aliquot of the supernatant $(30 \mu \mathrm{L}$ per well) and working assay solution were added to 96 -well plates, followed by incubation for $15 \min$ at $37{ }^{\circ} \mathrm{C}$. Separately, double distilled water and standard phenol solution were added as negative control (NC) and standard control. To quantify the 
ALP activity, the absorbance was measured at $520 \mathrm{~nm}$ using the SPECTROstar Nano microplate reader (BMG Labtech, Ortenberg, Germany).

The BCIP/NBT Alkaline Phosphatase Color Development Kit (Beyotime, Shanghai, China) was used to assess ALP activity. Like previously mentioned, cells were fixed with 4\% PFA, washed with PBS, and then stained with dye liquor. The stained plates were captured with a scanner and a light microscope (Olympus, Japan).

\section{Multipotent Differentiation Assay}

For experiments on multipotent differentiation, SCAPs were seeded in a specific differentiation medium. Osteogenicinducing medium (OIM) was supplemented with 10\% FBS (BioInd, Kibbutz, Israel), 10-8 mol/L dexamethasone, $50 \mathrm{mg} /$ $\mathrm{L}$ ascorbic acid, and $10 \mathrm{mmol} / \mathrm{L} \beta$-glycerophosphate (Sigma-Aldrich, St. Louis, MO, USA). Adipogenic differentiation medium was supplemented with $10 \% \mathrm{FBS}, 500 \mu \mathrm{mol} / \mathrm{L}$ 3-isobutyl-1-methylxanthine (IBMX), $1 \mu \mathrm{mol} / \mathrm{L}$ dexamethasone, $200 \mu \mathrm{mol} / \mathrm{L}$ indomethacin, and $10 \mu \mathrm{g} / \mathrm{mL}$ insulin (Sigma-Aldrich). SCAPs were seeded in 6-well culture plates for 21 days and the inducing medium was changed every 3 days. After induction for 21 days or 28 days, mineralization nodules were identified by Alizarin Red staining (Solarbio) and lipid droplets were identified by Oil Red O staining (Solarbio).

\section{Phenotype Identification}

For phenotype analysis, SCAPs (passages 3-5) were suspended and characterized with monoclonal antibodies reactive to CD34, CD44, CD45, CD90, and CD105 (eBioscience, San Diego, CA, USA), which incubated for $1 \mathrm{~h}$ on ice. PBS was used to wash SCAPs thoroughly and resuspend them in a single-cell suspension. After washing three times, samples were analyzed using a cell analyzer (Beckman Coulter, Brea, CA, USA).

\section{Generation of TAZ-Overexpression or $\beta$-Catenin-Overexpression SCAPs}

SCAPs were transfected with lentiviruses to promote the overexpression of TAZ or $\beta$-catenin packaged by Genechem Company (Shanghai, China). The empty vector was employed as the negative control. The cells were incubated in the culture media plus lentiviruses and HitransG P for $9 \mathrm{~h}$. To estimate whether the overexpression model was constructed successfully or not, transfection efficiency was evaluated by observation with a fluorescence microscope, mRNA, and protein.

\section{Small Interfering RNA Transfection}

Three different siRNA targeting ROR2 mRNA (siROR2-1\#, siROR2-2\#, and siROR2-3\#) and non-targeting control (siCTRL) were synthesized by GenePharma Company. The sequences of siRNA are listed in Supplementary Table 1. SCAPs were seeded on 6-well plates $24 \mathrm{~h}$ before siRNA transfection. Lipofectamine 3000 (Invitrogen, Inc., Carlsbad, CA, USA) were incubated with opti-mem (Gibco-BRL, Grand Island, NY, USA) ahead of the mixture between siRNA for ROR2 (150 pmol per well) or siCTRL (150 pmol per well) and opti-mem. The above solutions were mixed and incubated at room temperature for $15 \mathrm{~min}$. Then, they were dropwise added to $\alpha$-MEM supplemented with $10 \%$ FBS. After $24 \mathrm{~h}$, the medium was replaced with a fresh $10 \% \mathrm{FBS} / \alpha-\mathrm{MEM}$ or osteogenic differentiation medium.

\section{Alizarin Red Staining and Quantitative Analysis}

To assess the extent of osteogenic differentiation, SCAPs were washed with PBS and fixed with 4\% PFA after 21 days. The cells were then washed 3 times prior to the addition of $0.2 \%$ Alizarin Red S at pH 8.3 (Solarbio) for $1 \mathrm{~h}$. The stained plates were captured with a scanner and a light microscope. To quantify the mineralized matrix deposition, the precipitation was dissolved with $10 \%$ cetylpyridinium chloride (CPC; Solarbio) and the absorbance was measured at $562 \mathrm{~nm}$ using the SPECTROstar Nano microplate reader (BMG Labtech, Ortenberg, Germany).

\section{Quantitative Real-Time PCR}

Quantitative real-time PCR (qRT-PCR) was applied to detect gene expression. Total RNA was extracted by Trizol Reagent (TaKaRa, Shiga, Japan). cDNA was synthesized from $1 \mu \mathrm{g}$ total RNA using Primer Script ${ }^{\circledR}$ RT Reagent Kit (TaKaRa) as per the manufacturer's instruction. Qualitative RT-PCR was performed by SYBR ${ }^{\circledR}$ Premix Ex Taq ${ }^{\mathrm{TM}}$ 
(TaKaRa) with Roche 480, and each RNA sample was assayed in triplicate. For the relative quantitative analysis of the target gene, Cq values were obtained and calculated with the comparative CT method. ${ }^{26}$ The target gene transcript levels were normalized according to an endogenous reference gene (GAPDH). Primer sequences for ALP, Osterix, RUNX2, ROR2, $\beta$-catenin, TAZ, and GAPDH are shown in Supplementary Table 2.

\section{Western Blotting Analysis}

SCAPs were lysed by radioimmunoprecipitation assay lysis buffer (Solarbio) with $1 \%$ phenylmethylsulfonyl fluoride (PMSF; Solarbio) and 1\% phosphatase inhibitor cocktail (Boster, Hubei, China). Samples were centrifuged at 12,000 rpm for $15 \mathrm{~min}$ and the supernatant was collected. Protein concentrations were measured by BCA assay kit (Solarbio), which informed the loading volume of total proteins $(40 \mu \mathrm{g})$. Gels were transferred onto PVDF membranes (Millipore, Billerica, MA, USA). The membranes were blocked with 5\% skim milk and incubated with the following primary antibody at $4{ }^{\circ} \mathrm{C}$ overnight: ALP (Huabio, Hangzhou, China); RUNX2 (Abcam, Cambridge, UK); GSK, p-GSK, and TAZ (Cell Signaling Technology, Beverly, MA); and ROR2 and $\beta$-catenin (Santa Cruz Biotechnology, Santa Cruz, CA). Secondary antibodies, goat anti-rabbit and goat anti-mouse (Proteintech, Hubei, China), were selected referring to the instruction of the primary antibodies. Luminescent autoradiography and an enhanced chemiluminescence system were applied to visualize the membranes. GAPDH was used as an endogenous reference protein.

\section{Immunocytofluorescence Staining}

For immunocytofluorescent analysis, SCAPs were washed with PBS and then fixed with 4\% PFA for 15 min. After being pretreated by $0.5 \%$ Triton (Solarbio) in PBS for $30 \mathrm{~min}$, the cells were blocked with 5\% BSA in PBS for $2 \mathrm{~h}$, which was prior to the addition of primary antibodies: mouse monoclonal anti-ROR2 (Santa Cruz Biotechnology) at a dilution of 1:50 or CD24 (eBioscience) at a dilution of 1:100. Goat anti-mouse antibody (Proteintech) was used as a secondary antibody at a dilution of 1:100 and incubating time was about $1 \mathrm{~h}$. The cells were then washed three times with PBS and stained with DAPI to visualize the cell nuclei.

\section{Statistical Analysis}

All experiments were performed in triplicate. GraphPad Prism 8 (GraphPad Software, Inc., La Jolla, CA, USA) was applied to analyze the statistics. The data are expressed as mean \pm standard deviation. Statistical analysis was performed using Student's unpaired Student's $t$-test and one-way analysis of variance (ANOVA). The statistical significance was set at $\mathrm{P}$-value $<0.05$.

\section{Results}

\section{Isolation and Characterization of SCAPs}

Primary SCAPs (Figure 1B) separated from root apical papilla (Figure 1A) could be observed under a microscope after 7 days and passage 3 of SCAPs presented spindle-shaped morphology (Figure 1C). The limiting dilution technique was used to isolate SCAPs and SCAPs-formed colonies (Figure 1D) with 200 SCAPs in a dish $(6 \mathrm{~cm})$ within 7 days. To investigate multi-directional differentiation ability, cells were cultured in an osteogenic- and adipogenic-inducing medium. Several mineralization nodules were stained in Alizarin Red after 21 days cultured in the osteogenic-inducing medium (Figure 1E) and lipid droplets presenting Oil Red O-positive formed after 28 days cultured in the adipogenicinducing medium (Figure 1F). The expressions of CD34, CD44, CD45, CD90, and CD105 were analyzed by flow cytometry to characterize phenotypic markers of SCAPs. SCAPs expressed the MSC-related markers such as CD44, CD90, and CD105 (Figure 1H), but were negative for the hematopoietic markers such as CD34 and CD45 (Figure 1H). A specific surface marker of SCAPs, CD24, ${ }^{27}$ was detected by immunofluorescence, suggesting its positive expression (Figure 1G). These results indicated that SCAPs possess the characteristics of mesenchymal sources and can be used for further experiments. 
A

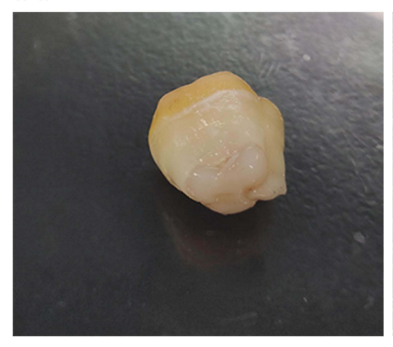

B

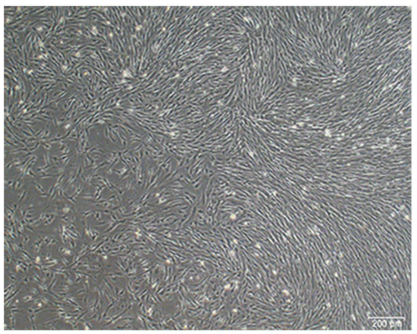

C

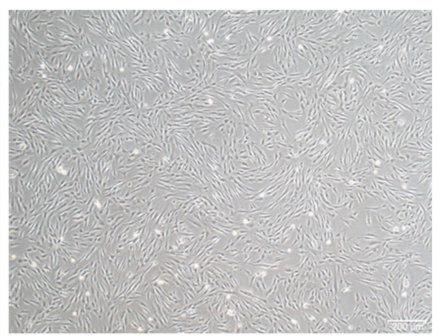

D

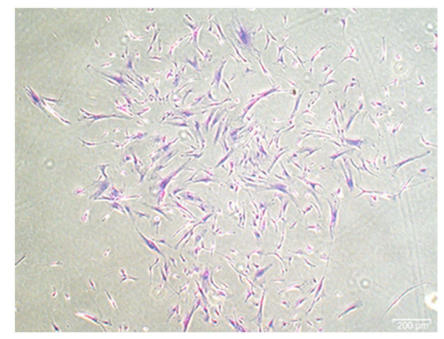

E

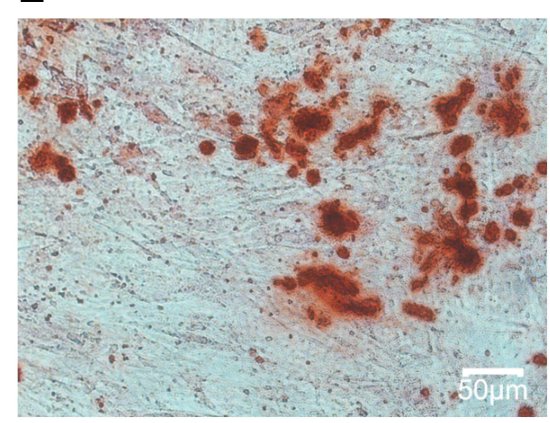

$\mathbf{F}$

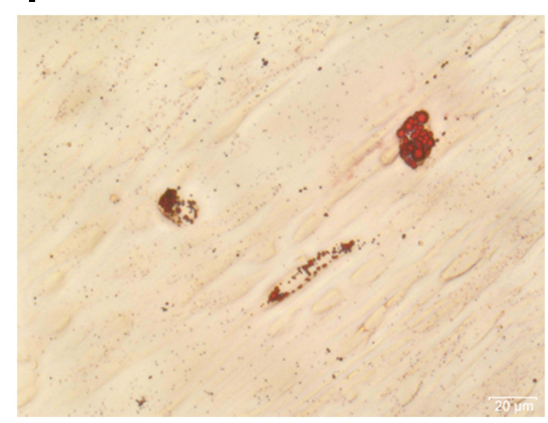

G

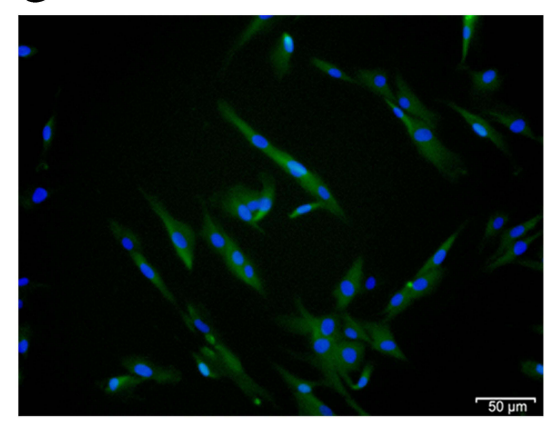

H
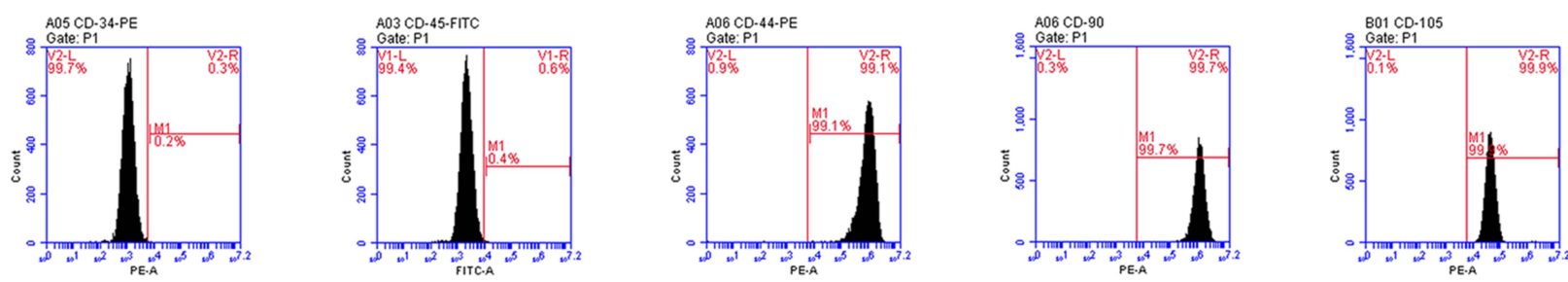

Figure I Isolation and characterization of SCAPs (A) Developing root apical papilla tissue of extracted human immature third molars. Scale bar: 200nm. (B) Primary SCAPs could be observed under a microscope after 7 days. Scale bar: $200 \mathrm{~nm}$. (C) SCAPs (passage 3) presented spindle-shaped morphology. Scale bar: 200nm. (D) Colony formation of cells were stained by crystal violet. Scale bar: $200 \mathrm{~nm}$. (E) Mineralization nodules stained by Alizarin red staining after osteogenic induction for $2 \mathrm{I}$ d. Scale bar: $50 \mathrm{~nm}$. (F) Lipid droplets stained by Oil Red O after adipogenic induction for $28 \mathrm{~d}$. Scale bar: 20nm. (G) SCAPs expressed CD24 positively by immunofluorescent microscopy. Scale bar: 50nm. (H) Phenotypic markers observed under flow cytometry. SCAPs were positive for CD44, CD90 and CDI05, but negative for CD34 and CD45.

\section{Wnt5a Suppresses Osteogenesis of SCAPs and TAZ Expression}

To examine the effects of Wnt5a on the osteogenesis of SCAPs, SCAPs were treated with increasing concentrations of Wnt5a (10, 50, and $100 \mathrm{ng} / \mathrm{mL})$ for 7 days. Quantitative RT-PCR demonstrated that the expression levels of bone-related genes, such as ALP, RUNX2, and TAZ, were significantly lower with Wnt5a (100 ng/mL) than cells cultured in OIM (Figure 2A and F). Therefore, $100 \mathrm{ng} / \mathrm{mL}$ was the dose applied to the rest of the experiments.

ALP has been considered a typical marker of the early osteogenic differentiation of stem cells. An ALP staining assay and ALP activity assay showed that Wnt5a $(100 \mathrm{ng} / \mathrm{mL})$ suppressed ALP expression (Figure $2 \mathrm{~B}$ and C). A similar conclusion was supported by Alizarin Red staining and quantitative analysis (Figure 2E), with much less mineralized nodule formation in the Wnt5a $(100 \mathrm{ng} / \mathrm{mL})$ group. Western blotting demonstrated that the expression levels of bonerelated genes such as ALP and RUNX2 decreased markedly under Wnt5a stimuli (Figure 2D). The above results suggest that Wnt5a inhibits the osteogenesis of SCAPs.

To explicate the effect of Wnt5a on TAZ-mediated osteogenesis in SCAPs, the expression of TAZ was examined under an osteogenic inducing medium with $100 \mathrm{ng} / \mathrm{mL}$ Wnt5a or without Wnt5a after 7 days. Quantitative RT-PCR confirmed that the levels of TAZ expression were markedly downregulated (Figure 2F). Western blotting results revealed the same outcome (Figure $2 \mathrm{G}$ ). Based on the above results, it can be concluded that Wnt5a suppresses TAZ during osteogenesis. 

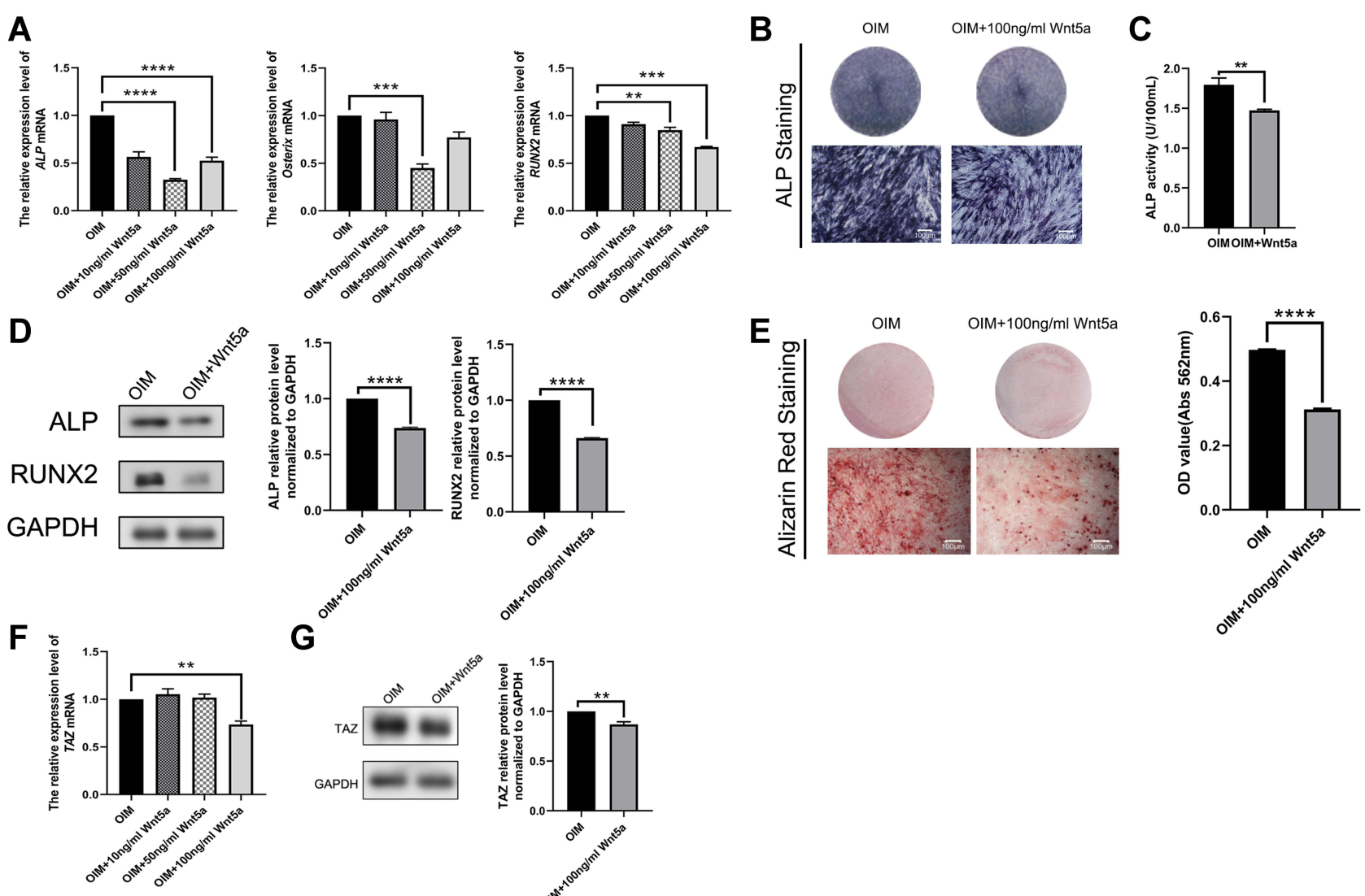

G
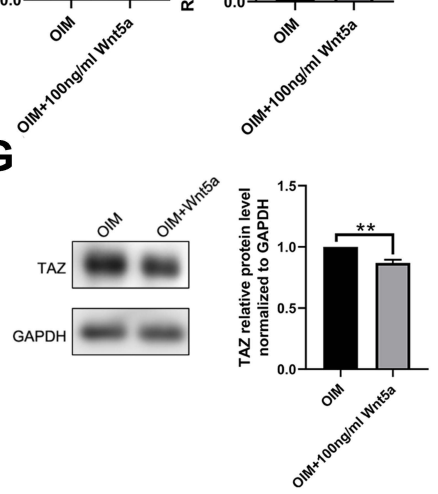

Figure 2 Effect of Wnt5a on the osteogenesis of SCAPs (A) Quantitative RT-PCR analysis of ALP, Osterix, RUNX2 and TAZ in SCAPs treated with increasing concentration of Wnt5a $(10,50,100 \mathrm{ng} / \mathrm{mL})$ for 7 days. (B) ALP staining of groups with or without $50 \mathrm{ng} / \mathrm{mL}$ at day 7 . (C) ALP activity of groups with or without $50 \mathrm{ng} / \mathrm{mL}$ at day 7 . (D) Western blotting analysis of ALP and RUNX2 after culturing in $50 \mathrm{ng} / \mathrm{mL} \mathrm{Wnt5a}$ for 7 days. (E) Alizarin red staining and semi-quantitative analysis of groups with or without $50 \mathrm{ng} / \mathrm{mL}$ at day 21 . (F) Quantitative RT-PCR analysis of TAZ in SCAPs treated with increasing concentration of Wnt5a (I0, 50, $100 \mathrm{ng} / \mathrm{mL})$ for $7 \mathrm{days}$. (G) Western blotting analysis of TAZ after culturing in $50 \mathrm{ng} / \mathrm{mL}$ Wnt5a for 7 days. Data are shown as means \pm SD.**P $<0.01, * * * \mathrm{P}<0.001$, $* * * * \mathrm{P}<0.0001$.

\section{TAZ Overexpression Promotes Osteogenesis of SCAPs}

To explore the relationship between TAZ and osteogenesis, lentivirus was transfected to overexpress TAZ in SCAPs. Transfection efficiency was evaluated by observation fluorescence microscope (Figure 3A), mRNA (Figure 3B), and protein (Figure 3C) levels of TAZ. The mRNA or protein level of TAZ was significantly upregulated in the OETAZ group compared with OENC.

After being cultured in OIM for 3 and 7 days, ALP staining and ALP activity assay showed that ALP was higher in OETAZ than in OENC (Figure 3E and F). A similar result was obtained by the measurement of mineralized nodules (Figure $3 \mathrm{G}$ ). In addition, the mRNA and protein levels of bone-related factors were detected, and they were more markedly expressed in OETAZ than in OENC (Figure 3D and H). Therefore, TAZ overexpression SCAPs showed stronger osteogenic potential than OENC SCAPs.

\section{Wnt5a Inhibits TAZ-Mediated Osteogenesis in SCAPs}

To further confirm the relationship between Wnt5a and TAZ-mediated osteogenesis, OENC and OETAZ were stimulated with Wnt5a for 7 days. Lighter ALP staining (Figure 4C), lower ALP activity assay (Figure 4D) and fewer mineralized nodules (Figure 4E) were observed in the OETAZ plus Wnt5a group compared to the OETAZ group. Similarly, levels of bone-related factors were also downregulated in the OETAZ plus Wnt5a group compared with the OETAZ group (Figure 4A and B). In summary, Wnt5a inhibits TAZ-mediated osteogenesis in SCAPs. 


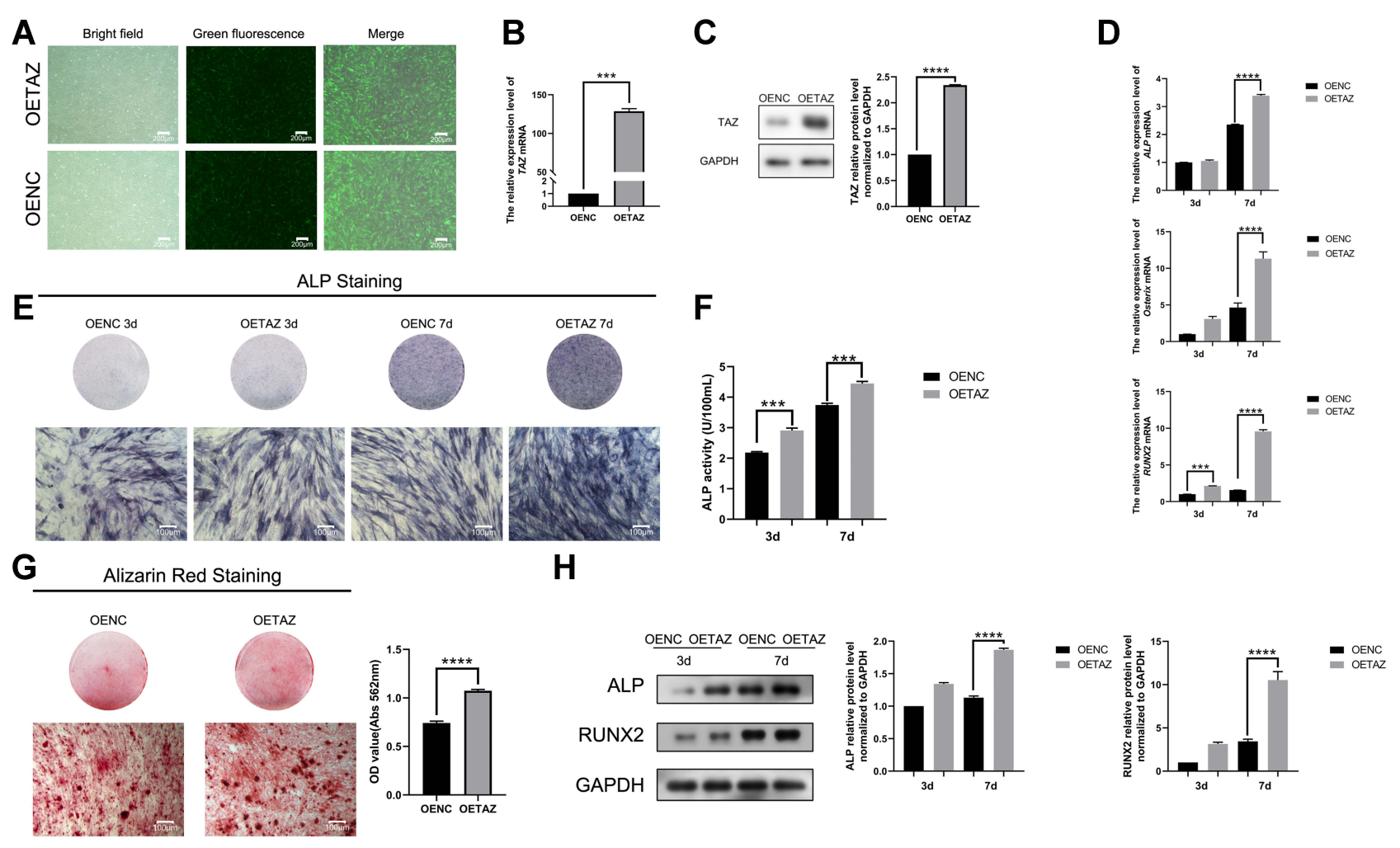

Figure 3 TAZ overexpression promotes osteogenesis of SCAPs (A-C) Transfection efficiency was evaluated by observation fluorescence microscope, mRNA, and protein levels of TAZ. (D) Quantitative RT-PCR analysis of ALP, Osterix, RUNX2 in TAZ-overexpression SCAPs (OETAZ) and corresponding control group (OENC) for 3 days and 7 days. (E) ALP staining of OENC and OETAZ for 3 days and 7 days. (F) ALP activity of OENC and OETAZ for 3 days and 7 days. (G) Alizarin red staining and quantitative analysis of OENC and OETAZ for 21 days. $(\mathbf{H})$ Western blotting analysis of ALP and RUNX2 in OENC and OETAZ for 3 days and 7 days. Data are shown as means \pm SD. $* * * \mathrm{P}<0.001$, $* * * * \mathrm{P}<0.0001$.

\section{ROR2 Transported Wnt5a to Affect TAZ Mediated Osteogenesis in SCAPs}

To investigate crosstalk between the noncanonical Wnt pathway and TAZ, the expression of ROR2, a receptor of the noncanonical Wnt pathway, was examined. ROR2 was positive in human SCAPs (Figure 5A) stained by immunocytofluorescence, while a control group stained with normal mouse IgG was negative in SCAPs. Afterward, the ROR2 knockdown model was constructed by ROR2 siRNA. The knockdown efficiency of siROR2\#1 was the optimal sequence as evaluated by RT-PCR and western blotting (Figure 5B and C). This sequence was used for the remainder of the experiment. The expression of ALP, RUNX2 and Osterix protein was upregulated in the ROR2 siRNA plus Wnt5a group when compared with siROR2 (Figure 5D), indicating that ROR2 transported Wnt5a to affect TAZ-mediated osteogenesis.

\section{Relationship Between Wnt5a and Canonical Wnt Pathway on TAZ-Mediated Osteogenesis}

The canonical Wnt pathway-related intracellular signaling molecules in SCAPs were examined to gain insight into the mechanism of the canonical Wnt pathway on Wnt5a/TAZ mediated osteogenesis. The expressions of $\beta$-catenin, p-GSK3 $\beta$, and TAZ were lower in the ROR2 siRNA plus Wnt5a group than the ROR2 siRNA group, whereas the total GSK-3 $\beta$ was nearly unchanged in the ROR2 siRNA plus Wnt5a group (Figure 6A). Based on the above results, it can be concluded that Wnt5a/ROR2 suppresses TAZ with a canonical Wnt pathway.

\section{$\beta$-Catenin is Regular for Osteogenesis via the Wnt5a/ROR2/TAZ Pathway}

Lentivirus was transfected to overexpress $\beta$-catenin in SCAPs to determine whether Wnt5a regulates the canonical Wnt pathway to suppress TAZ. Transfection efficiency was evaluated by observation fluorescence microscope (Figure 7A), 
A
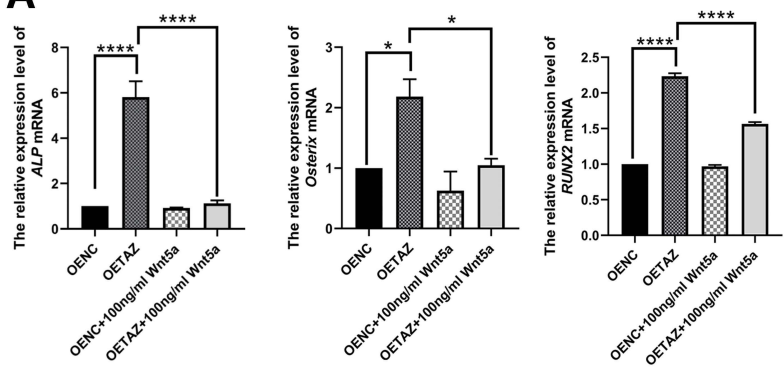

C

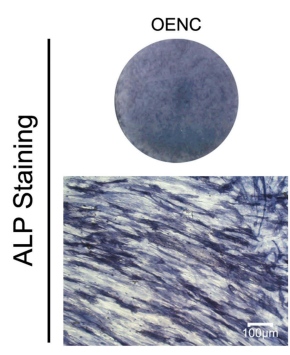

E

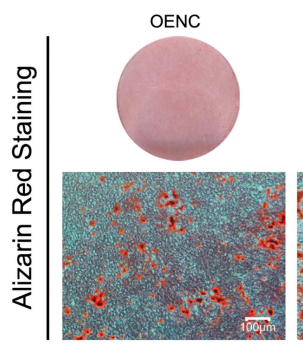

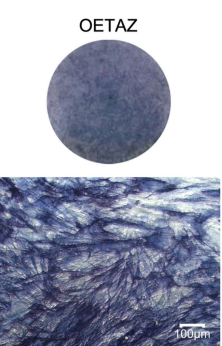

OENC+100ng/ml Wnt5a
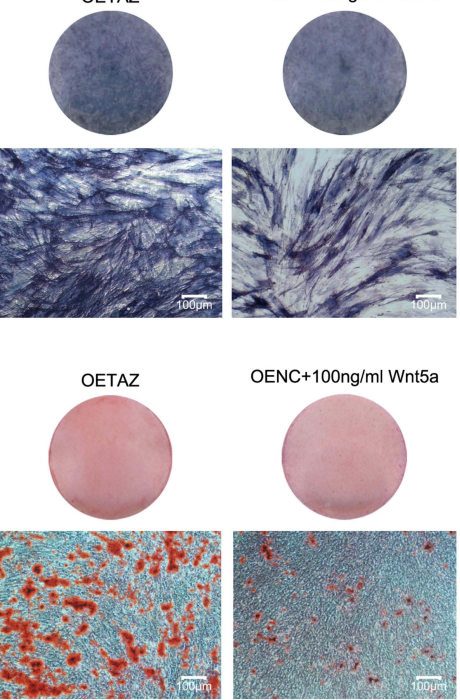

B

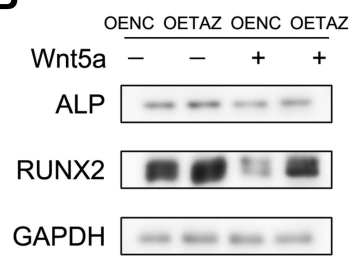

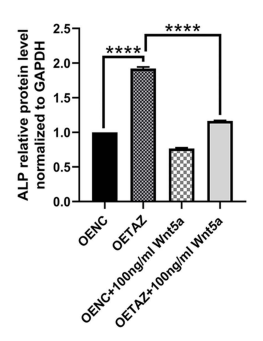

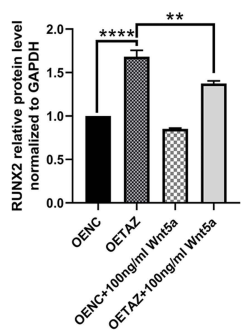

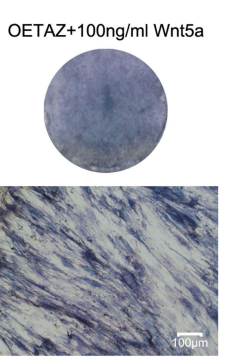

D
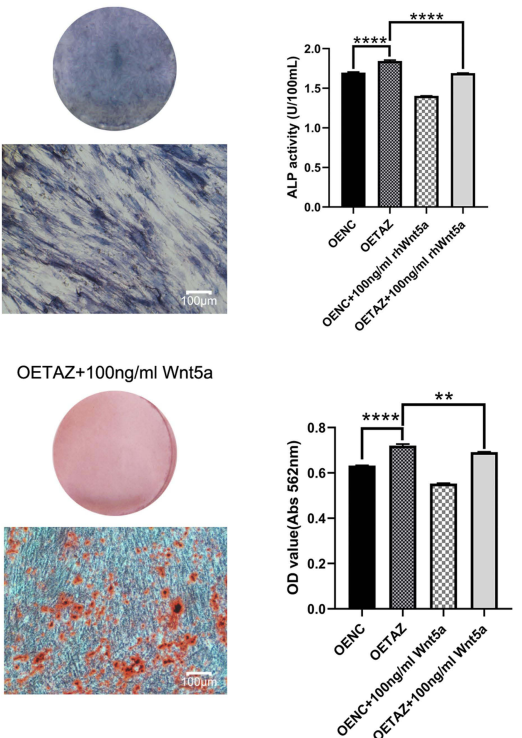

Figure 4 Effects of Wnt5a on TAZ mediated osteogenesis in SCAPs (A) Quantitative RT-PCR analysis of ALP, Osterix and RUNX2 in OENC, OETAZ, OENC plus Wnt5a and OETAZ plus Wnt5a group for 7 days. (B) Western blotting analysis of ALP and RUNX2 in OENC, OETAZ, OENC plus Wnt5a and OETAZ plus Wnt5a group for 7 days. (C) ALP staining of OENC, OETAZ, OENC plus Wnt5a and OETAZ plus Wnt5a group for 7 days. (D) ALP activity of OENC, OETAZ, OENC plus Wnt5a and OETAZ plus Wnt5a group for 7 days. (E) Alizarin red staining and quantitative analysis of OENC, OETAZ, OENC plus Wnt5a and OETAZ plus Wnt5a group for 21 days. Data are shown as means $\pm S D$. $* P<0.05, * * P<0.01, * * * * P<0.0001$

mRNA a (Figure 7B), and the protein levels of TAZ (Figure 7C). The mRNA or protein level of TAZ was significantly upregulated in the OE $\beta$-catenin group compared with OENC.

Further investigation revealed that the TAZ expression of OE $\beta$-catenin was much higher than OENC, whereas TAZ expression was dominantly downregulated by OE $\beta$-catenin cultured with Wnt5a in comparison to the $\beta$-catenin group (Figure 7H-I). Lighter ALP staining, lower ALP activity assay and fewer mineralized nodules, and less expression of bone-related proteins were also observed in OE $\beta$-catenin plus Wnt5a (Figure 7D-G). All of the above suggested that $\beta$ catenin regulates TAZ-mediated osteogenesis and there may be a Wnt5a/ROR2/ $\beta$-catenin/TAZ pathway that regulates the osteogenesis of SCAPs.

\section{Discussion}

Regeneration-based therapy has been a hot issue in modern medicine and seed cells are at its core. SCAPs are promising cells in the field of regenerative medicine. ${ }^{28}$ SCAPs isolated and cultured from immature tooth roots possess the properties of self-renewal, proliferation, migration, and multidirectional differentiation. ${ }^{28,29}$ Furthermore, SCAPs possess low immunogenicity and are capable of suppressing T cell proliferation. ${ }^{28,30} \mathrm{CD} 24$ is a specific marker to distinguish SCAPs from dental pulp cells. ${ }^{27}$ A high percentage of CD24-expressing SCAPs exhibit osteogenic differentiation 
A

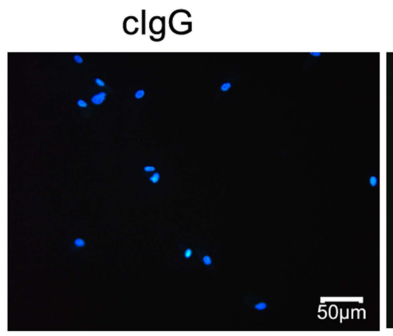

B

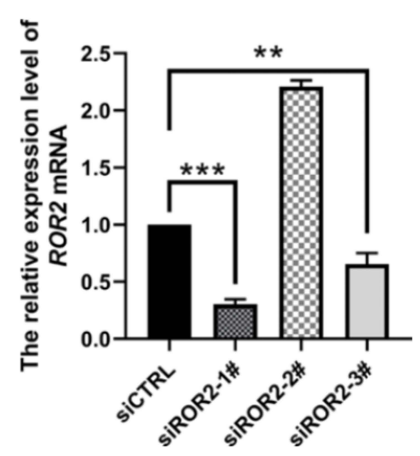

C

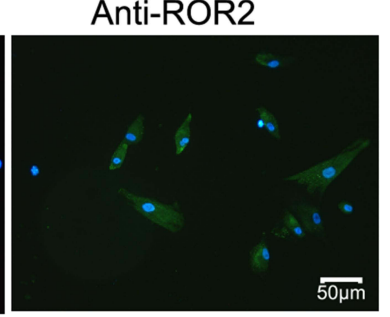

ROR2

GAPDH
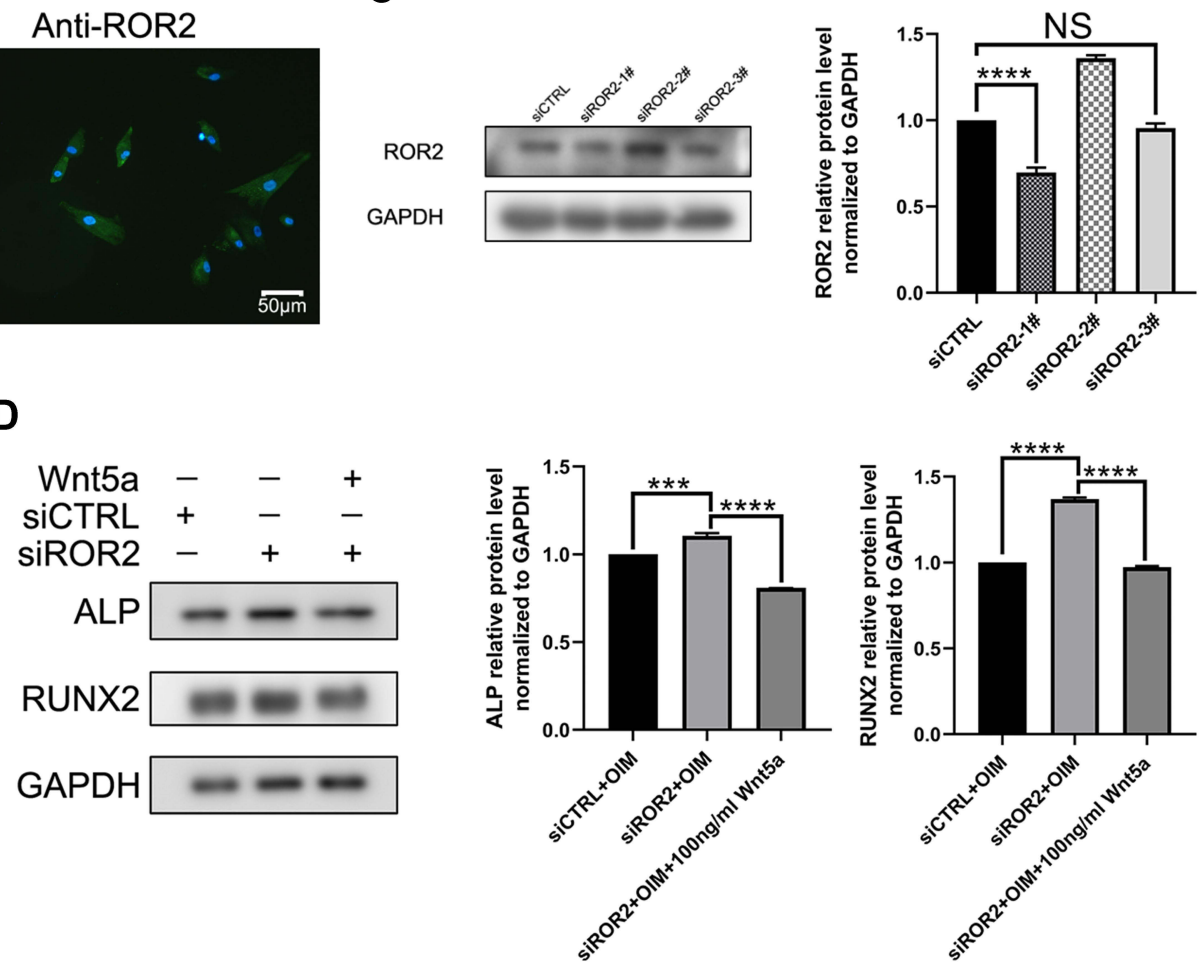

Figure 5 Effects of Wnt5a/ROR2 on TAZ mediated osteogenesis in SCAPs (A) SCAPs expressed ROR2 positively by immunofluorescent microscopy. Control group stained with mouse control $\operatorname{lgG}(\mathrm{clgG})$ was negative in SCAPs. Scale bar: $50 \mathrm{~nm}$. (B-C) Transfection efficiency was evaluated by mRNA, and protein levels of three siROR2. mRNA was collected after $48 \mathrm{~h}$, and protein was collected after $72 \mathrm{~h}$. (D) Western blotting analysis of ALP and RUNX2 in siCTRL, siROR2 and siROR2 plus Wnt5a group for 3 days. Data are shown as means $\pm \mathrm{SD}$. $* * \mathrm{P}<0.01$, $* * * \mathrm{P}<0.001$, $* * * * \mathrm{P}<0.0001$.

Abbreviation: NS, no significance.

capacity. ${ }^{27}$ In this study, CD24 on SCAPs was examined by immunofluorescence and cells showed a high percentage of expression, which hinted at potential osteogenic differentiation capacity.

TAZ is a core effector of the Hippo pathway and combines with TEAD-family DNA-binding transcription factors to control gene expression in response to the Hippo pathway off, which is involved in proliferation, migration, differentiation, and cell junction contact. ${ }^{31-34}$ The YAP/TAZ depletion model has been found to decrease bone accrual and reduce intrinsic bone material properties through impaired collagen content and organization. ${ }^{35}$ At the cellular level in vivo, YAP/TAZ ablation reduced osteoblast activity and increased osteoclast activity, in an allele dose-dependent manner, impairing bone accrual and remodeling. ${ }^{35}$ At the cellular level in vitro, increasing evidence confirmed that TAZ promotes osteogenic differentiation. ${ }^{9,36-38}$ Hao $\mathrm{J}$ and others found TAZ translocates into the nucleus and promotes osteogenesis of bone marrow mesenchymal stem cells (BMSCs) through activating RUNX2. ${ }^{36}$ Tan FZ and others found TAZ promotes the osteogenic differentiation of mesenchymal stem cells in the rat model of osteoporosis by repressing the PI3K/Akt signaling. ${ }^{37}$ In the present study, we conclude that TAZ promotes osteogenic differentiation of SCAPs, expressing upregulated bone-related factors, dark ALP staining, and numerous mineralized nodules.

Noncanonical Wnt pathway is independent of LRPs and $\beta$-catenin and complex with Frizzled (Fzd) and the receptor tyrosine kinase-like orphan receptor 2 (ROR2) to activate downstream signaling responses, such as the Ca2+/NFAT pathway in tumor and kidney cells, as well as osteoblasts, and the planar cell polarity (PCP)/convergent extension (CE) pathway, which involves JNK and so on. ${ }^{39-41} \mathrm{Wnt} 5 \mathrm{a}$ is a representative ligand of noncanonical Wnt pathway and exhibit potentiality on stemness maintenance and osteogenesis of MSCs ex vivo. ${ }^{15}$ Loss-of-function mutations in Wnt5a and ROR2 cause autosomal recessive Robinow syndrome, characterized by midfacial hypoplasia, limb bone shortening, and genital abnormalities. ${ }^{17,42,43}$ ROR2 is a member of the ROR family of receptor tyrosine-protein kinases and mediates diverse noncanonical Wnt5a signaling pathways such as Wnt/Ca2+ and Wnt/planar cell polarity pathways. ${ }^{15,40,44,45}$ In 
A

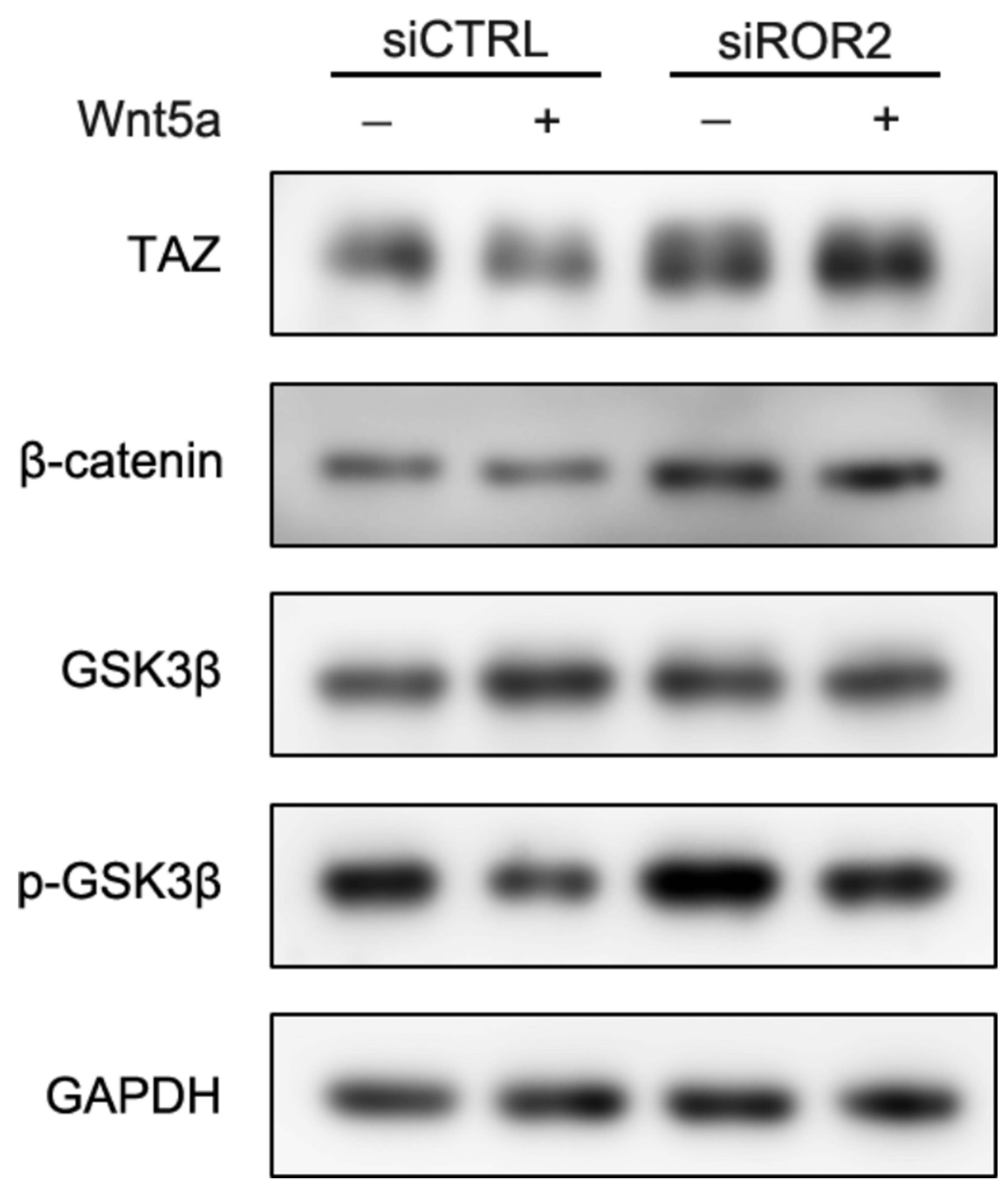

Figure 6 Relationship between Wnt5a and canonical Wnt pathway on TAZ-mediated osteogenesis (A) Western blotting analysis of $\beta$-catenin, GSK3 $\beta$, p-GSK3 $\beta$ and TAZ in siCTRL, siROR2, siCTRL plus Wnt5a and siROR2 plus Wnt5a group for 3 days.

addition, it also inhibits the $\beta$-catenin-T cell factor/lymphoid enhancer factor pathway. ${ }^{45}$ Previous research found Wnt5a/ ROR2 signaling is involved in the BMP-2-mediated osteoblast differentiation in a Smad-independent pathway. ${ }^{18}$ It also enhances receptor activation of nuclear factor- $\mathrm{KB}$ (RANK) expression in osteoclast precursors by activating JNK and recruiting c-Jun on the promoter of the gene encoding RANK, thereby enhancing RANK ligand (RANKL)-induced osteoclastogenesis. ${ }^{18}$ In our study, our findings demonstrate that Wnt5a suppressed osteogenesis in SCAPs and 100ng/mL is the optimal concentration chosen for the remainder of the experiment. ROR2 knockdown partly reverses suppression of Wnt5a on the expression of bone-related factors such as ALP, RUNX2, and Osterix. This result indicates that ROR2 participates in the process preventing osteogenic differentiation of SCAPs. A similar conclusion has even been made that adipocyte-derived MVs-miR-148a promoted adipogenic differentiation and suppressed osteogenic differentiation via targeting the Wnt5a/ROR2 pathway. ${ }^{46}$ Moreover, Wnt5a/ROR2/JNK signaling was clarified as a negative regulator of mineralization, preventing the development of non-physiological mineralization in periodontal ligament tissue. ${ }^{17}$

Recently, cross-talk between Wnt signaling and Hippo signaling has attracted widespread attention. However, interactions between noncanonical Wnt pathway and YAP/TAZ have been rarely referred. Park and others discovered that treatment with Wnt5a in MCF10A mammary epithelial cells showed accumulation of YAP/TAZ as well as 
A
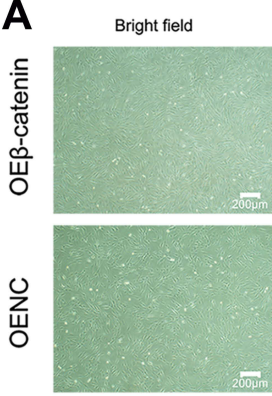

D

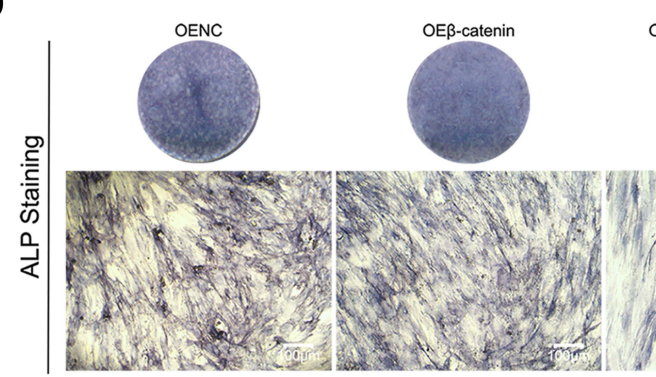

F
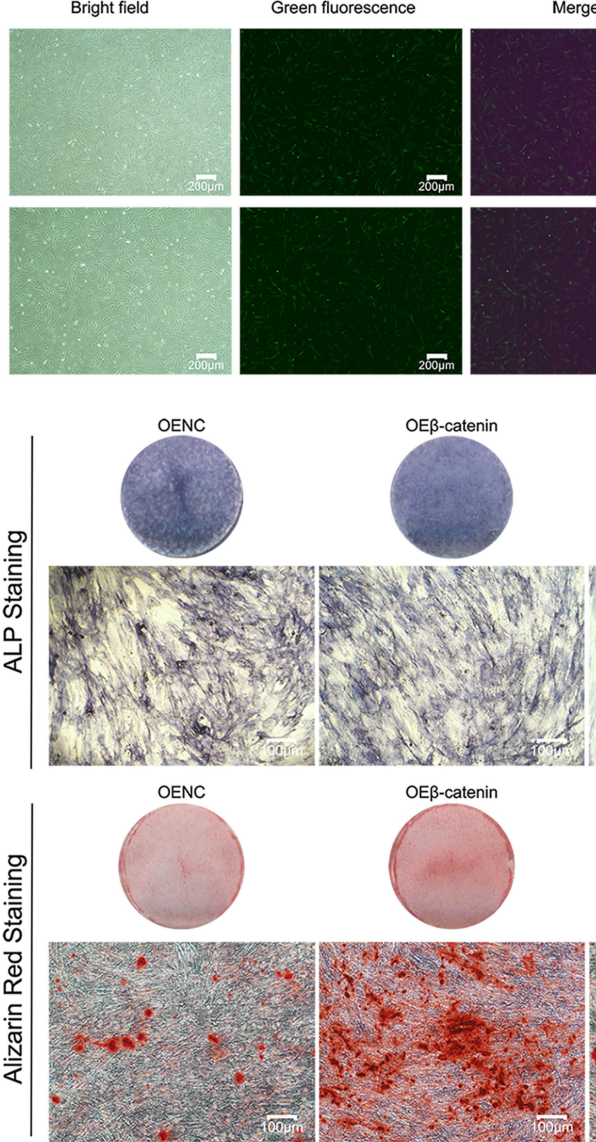

B

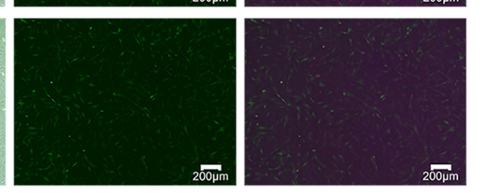

OENC+100ng/ml Wnt5a
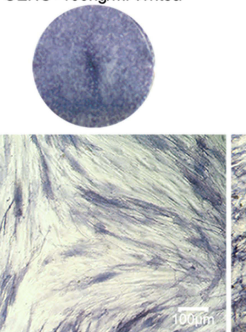

OENC+100ng/ml Wnt5a
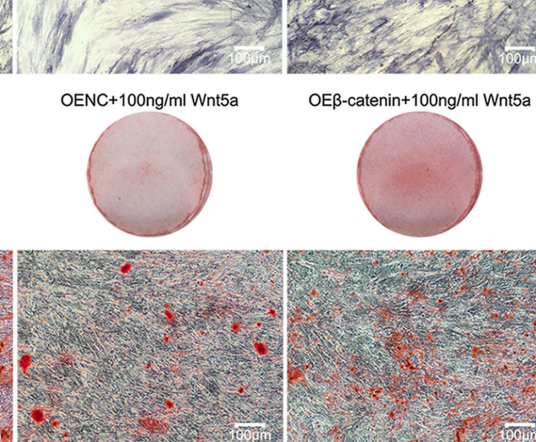

OEß-catenin+100ng/ml Wnt5a
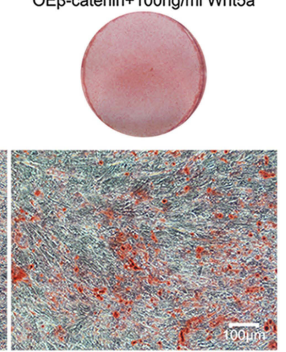

G

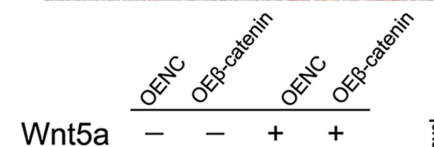

ALP

\section{$----$}

RUNX2

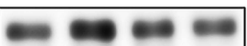

GAPDH

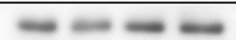

H

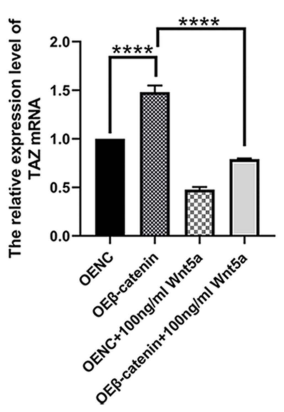

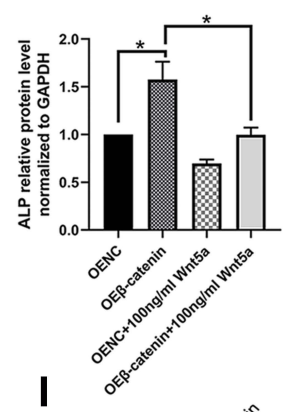
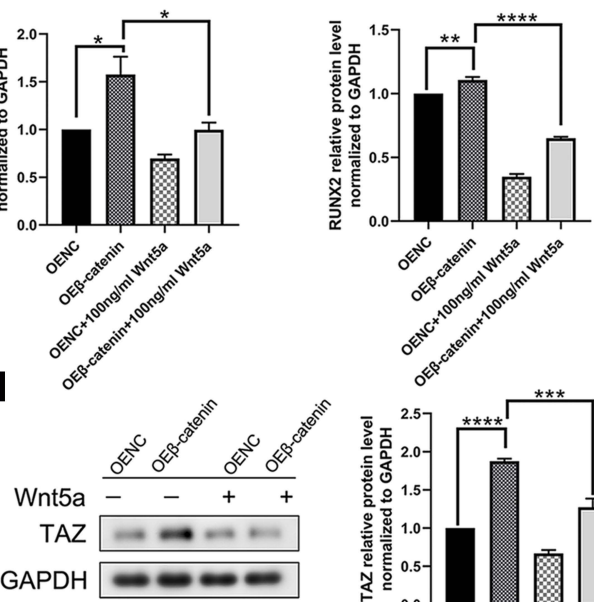

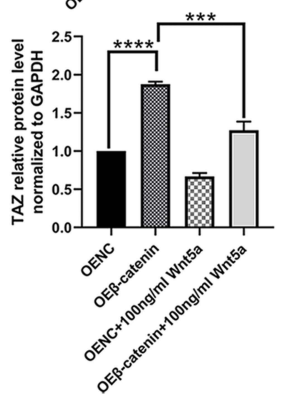

C

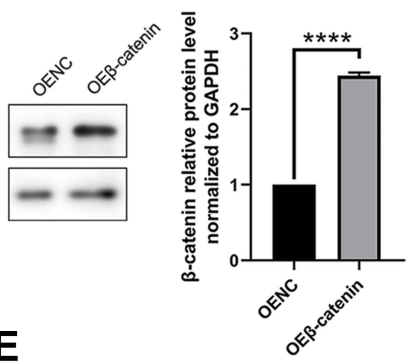

E
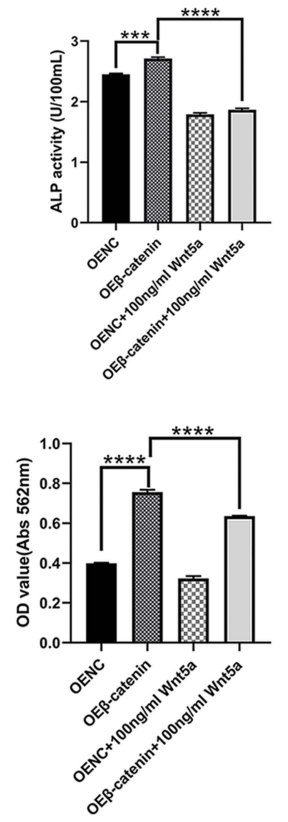

Figure $7 \beta$-catenin is regular for osteogenesis via Wnt5a/ROR2/TAZ pathway (A-C) Transfection efficiency was evaluated by observation fluorescence microscope, mRNA, and protein levels of $\beta$-catenin. (D) ALP staining of OENC, OE $\beta$-catenin, OENC plus Wnt5a and OE $\beta$-catenin plus Wnt5a for 7 days. (E) ALP activity of OENC, OE $\beta$ catenin, OENC plus Wnt5a and OE $\beta$-catenin plus Wnt5a for 7 days. (F) Alizarin red staining and quantitative analysis of Alizarin red staining of OENC, OE $\beta$-catenin, OENC plus Wnt5a and OE $\beta$-catenin plus Wnt5a group for 21 days. (G) Western blotting analysis of ALP and RUNX2 in OENC, OE $\beta$-catenin, OENC plus Wnt5a and OE $\beta$-catenin plus Wnt5a group for 7 days. (H-I) Quantitative RT-PCR analysis and Western blotting analysis of TAZ in OENC, OE $\beta$-catenin, OENC plus Wnt5a and OE $\beta$-catenin plus Wnt5a group for 7 days. Data are shown as means \pm SD. $* \mathrm{P}<0.05$, **P $<0.01$, ***P $<0.00$ I, ****P $<0.000$ I. 
downstream gene CTGF and CYR61 expression. ${ }^{22}$ In the present study, we notice that Wnt5a inhibits TAZ expression and TAZ-mediated osteogenesis. Knockdown of ROR2 can reverse TAZ expression and upregulate ALP, RUNX2, and Osterix. This result indicates the noncanonical Wnt pathway suppresses Hippo/TAZ mediated osteogenic differentiation, implying the existence of a Wnt5a/ROR2/TAZ axis. Interestingly, cross-talk between canonical Wnt signaling and Hippo signaling presents opposite conclusion. In Wnt-ON cells, YAP/TAZ accumulates in the nucleus and activates Wnt/YAP/ TAZ-dependent biological functions such as osteogenic differentiation. In Wnt OFF cells, YAP/TAZ is critical for $\beta$ catenin degradation, and depletion of YAP/TAZ leads to the activation of $\beta$-catenin/TCF transcriptional responses. ${ }^{19,38,47}$ Moreover, Wnt3a promotes the dephosphorylation and nuclear localization of TAZ via PP1A, which stabilizes TAZ and prevents it from binding 14-3-3 proteins. ${ }^{46}$ TAZ stimulated by Icariin could be blocked by DKK1, an antagonist of the canonical Wnt pathway, indicating an interaction of the Wnt/TAZ pathway. ${ }^{48}$ Based on the above, we wondered if there might be a connection between Wnt5a/ROR2 and canonical Wnt pathway on TAZ expression. And thus, we detected canonical Wnt signaling pathway molecules such as GSK3 $\beta$, phosphorylated GSK3 $\beta$, and $\beta$-catenin. ROR2 knockdown cultured with the Wnt5a group showed lower $\beta$-catenin, TAZ, and higher phosphorylation of GSK-3 $\beta$ than the ROR2 knockdown group. Therefore, we propose that Wnt5a/ROR2 downregulates TAZ via antagonism to the canonical Wnt pathway. To further elucidate the mechanism, we constructed a $\beta$-catenin overexpression model in vitro. The results of the present study show that $\beta$-catenin overexpression upregulates the TAZ gene and partly prevents suppression of Wnt5a, which demonstrates that the noncanonical Wnt pathway regulates the Hippo pathway partly through the canonical Wnt pathway in osteogenesis.

In summary, the present study verified that Wnt5a, the representative ligand of the noncanonical Wnt pathway, suppressed the osteogenesis of SCAPs and TAZ expression. Moreover, TAZ, a core regulator of the Hippo pathway, promoted osteogenesis of SCAPs and was downregulated when SCAPs were cultured with Wnt5a. Further research confirmed that the typical receptor of the noncanonical Wnt pathway, ROR2, could reverse the suppression effect of Wnt5a on both bone-related factors and TAZ. And the canonical Wnt pathway was identified as a participant in Wnt5a/ ROR2/TAZ mediated osteogenesis, demonstrating that there may be a Wnt5a/ROR2/ $\beta$-catenin/TAZ pathway that regulates the osteogenesis of SCAPs.

\section{Conclusion}

Taken together, the present study demonstrates that noncanonical Wnt5a/ROR2 signaling suppresses TAZ-mediated osteogenesis of SCAPs. And the process is regulated by canonical Wnt signaling molecules such as GSK3 $\beta$, phosphorylated GSK3 $\beta$, and $\beta$-catenin. In conclusion, noncanonical Wnt5a signaling suppresses Hippo/TAZ-mediated osteogenesis partly through the canonical Wnt pathway in SCAPs.

\section{Abbreviations}

SCAP, stem cells from the apical papilla; TAZ, transcriptional co-activator with PDZ-binding motif; ROR2, receptor tyrosine kinase-like orphan receptor 2; RANK, receptor activation of nuclear factor- $\kappa B$; RANK ligand, RANKL; FBS, fetal bovine serum; PFA, paraformaldehyde; PBS, phosphate buffer saline; OIM, osteogenic-inducing medium; IBMX, 3isobutyl-1-methylxanthine; siCTRL, non-targeting control; CPC, cetylpyridinium chloride; qRT-PCR, quantitative realtime PCR; PMSF, phenylmethylsulfonyl fluoride; BMSC, bone marrow mesenchymal stem cells; Fzd, Frizzled; PCP, planar cell polarity; ALP, alkaline phosphatase; RUNX2, runt-related transcription factor 2; GAPDH, glyceraldehyde-3phosphate dehydrogenase; BCA, bicinchoninic acid assay.

\section{Acknowledgments}

This work was supported by the Province Natural Science Foundation of Shandong Province, China (Grant No. ZR2019MH113).

\section{Disclosure}

The authors report no conflicts of interest in this work. 


\section{References}

1. Yang C, Li X, Sun L, Guo W, Tian W. Potential of human dental stem cells in repairing the complete transection of rat spinal cord. $J$ Neural Eng. 2017;14(2):026005. doi:10.1088/1741-2552/aa596b

2. Wang Y, Pang X, Wu J, et al. MicroRNA hsa-let-7b suppresses the odonto/osteogenic differentiation capacity of stem cells from apical papilla by targeting MMP1. J Cell Biochem. 2018;119(8):6545-6554. doi:10.1002/jcb.26737

3. Huang GT, Gronthos S, Shi S. Mesenchymal stem cells derived from dental tissues vs. those from other sources: their biology and role in regenerative medicine. J Dent Res. 2009;88(9):792-806. doi:10.1177/0022034509340867

4. Johnson R, Halder G. The two faces of Hippo: targeting the Hippo pathway for regenerative medicine and cancer treatment. Nat Rev Drug Discov. 2014;13(1):63-79. doi:10.1038/nrd4161

5. Kovar H, Bierbaumer L, Radic-Sarikas B. The YAP/TAZ pathway in osteogenesis and bone sarcoma pathogenesis. Cells. $2020 ; 9(4): 972$. doi:10.3390/cells 9040972

6. Byun MR, Jeong H, Bae SJ, Kim AR, Hwang ES, Hong JH. TAZ is required for the osteogenic and anti-adipogenic activities of kaempferol. Bone. 2012;50(1):364-372. doi:10.1016/j.bone.2011.10.035

7. Wang N, Li Y, Li Z, Liu C, Xue P. Sal B targets TAZ to facilitate osteogenesis and reduce adipogenesis through MEK-ERK pathway. $J$ Cell Mol Med. 2019;23(5):3683-3695. doi:10.1111/jcmm.14272

8. Xue P, Wu X, Zhou L, et al. IGF1 promotes osteogenic differentiation of mesenchymal stem cells derived from rat bone marrow by increasing TAZ expression. Biochem Biophys Res Commun. 2013;433(2):226-231. doi:10.1016/j.bbrc.2013.02.088

9. Wei Q, Holle A, Li J, et al. BMP-2 signaling and mechanotransduction synergize to drive osteogenic differentiation via YAP/TAZ. Adv Sci (Weinh). 2020;7(15):1902931. doi:10.1002/advs.201902931

10. Zhang B, Sun BY, Ji YW, et al. Expression and localization of Yap and Taz during development of the mandibular first molar in rats. Biotech Histochem. 2017;92(3):212-221. doi:10.1080/10520295.2016.1267799

11. Li CY, Hu J, Lu H, et al. $\alpha$ E-catenin inhibits YAP/TAZ activity to regulate signalling centre formation during tooth development. Nat Commun. 2016;7:12133. doi:10.1038/ncomms12133

12. Visweswaran M, Pohl S, Arfuso F, et al. Multi-lineage differentiation of mesenchymal stem cells - To Wnt, or not Wnt. Int J Biochem Cell Biol. 2015;68:139-147. doi:10.1016/j.biocel.2015.09.008

13. Nakanishi R, Akiyama H, Kimura H, et al. Osteoblast-targeted expression of Sfrp4 in mice results in low bone mass. J Bone Miner Res. 2008;23 (2):271-277. doi:10.1359/jbmr.071007

14. Maeda K, Kobayashi Y, Koide M, et al. The regulation of bone metabolism and disorders by Wnt signaling. Int J Mol Sci. 2019;20(22):5525. doi:10.3390/ijms20225525

15. Green JL, Kuntz SG, Sternberg PW. Ror receptor tyrosine kinases: orphans no more. Trends Cell Biol. 2008;18(11):536-544. doi:10.1016/j. tcb.2008.08.006

16. Lin M, Li L, Liu C, et al. Wnt5a regulates growth, patterning, and odontoblast differentiation of developing mouse tooth. Dev Dyn. 2011;240 (2):432-440. doi:10.1002/dvdy.22550

17. Nemoto E, Ebe Y, Kanaya S, et al. Wnt5a signaling is a substantial constituent in bone morphogenetic protein-2-mediated osteoblastogenesis. Biochem Biophys Res Commun. 2012;422(4):627-632. doi:10.1016/j.bbrc.2012.05.039

18. Maeda K, Kobayashi Y, Udagawa N, et al. Wnt5a-Ror2 signaling between osteoblast-lineage cells and osteoclast precursors enhances osteoclastogenesis. Nat Med. 2012;18(3):405-412. doi:10.1038/nm.2653

19. Azzolin L, Panciera T, Soligo S, et al. YAP/TAZ incorporation in the $\beta$-catenin destruction complex orchestrates the Wnt response. Cell. 2014;158 (1):157-170. doi:10.1016/j.cell.2014.06.013

20. Li N, Lu N, Xie C. The Hippo and Wnt signalling pathways: crosstalk during neoplastic progression in gastrointestinal tissue. FEBS J. 2019;286 (19):3745-3756. doi:10.1111/febs.15017

21. Byun MR, Hwang JH, Kim AR, et al. Canonical Wnt signalling activates TAZ through PP1A during osteogenic differentiation. Cell Death Differ. 2014;21(6):854-863. doi:10.1038/cdd.2014.8

22. Park HW, Kim YC, Yu B, et al. Alternative Wnt signaling activates YAP/TAZ. Cell. 2015;162(4):780-794. doi:10.1016/j.cell.2015.07.013

23. Feng Y, Liang Y, Zhu X, et al. The signaling protein Wnt5a promotes TGF $\beta 1$-mediated macrophage polarization and kidney fibrosis by inducing the transcriptional regulators Yap/Taz. J Biol Chem. 2018;293(50):19290-19302. doi:10.1074/jbc.RA118.005457

24. Arge S, Boldsen JL, Wenzel A, Holmstrup P, Jensen ND, Lynnerup N. Third molar development in a contemporary Danish 13-25 year old population. Forensic Sci Int. 2018;289:12-17. doi:10.1016/j.forsciint.2018.05.005

25. Ruparel NB, de Almeida JF, Henry MA, Diogenes A. Characterization of a stem cell of apical papilla cell line: effect of passage on cellular phenotype. J Endod. 2013;39(3):357-363. doi:10.1016/j.joen.2012.10.027

26. Schmittgen TD, Livak KJ. Analyzing real-time PCR data by the comparative C(T) method. Nat Protoc. 2008;3(6):1101-1108. doi:10.1038/ nprot.2008.73

27. Aguilar P, Lertchirakarn V. Comparison of stem cell behaviors between indigenous high and low-CD24 percentage expressing cells of stem cells from apical papilla (SCAPs). Tissue Cell. 2016;48(5):397-406. doi:10.1016/j.tice.2016.08.008

28. Kang J, Fan W, Deng Q, He H, Huang F. Stem cells from the apical papilla: a promising source for stem cell-based therapy. Biomed Res Int. 2019;2019:6104738. doi:10.1155/2019/6104738

29. Sonoyama W, Liu Y, Fang D, et al. Mesenchymal stem cell-mediated functional tooth regeneration in swine. PLoS One. 2006;1(1):e79. doi:10.1371/journal.pone.0000079

30. Ding G, Liu Y, An Y, et al. Suppression of T cell proliferation by root apical papilla stem cells in vitro. Cells Tissues Organs. 2010;191(5):357-364. doi:10.1159/000276589

31. Hansen CG, Moroishi T, Guan KL. YAP and TAZ: a nexus for Hippo signaling and beyond. Trends Cell Biol. 2015;25(9):499-513. doi:10.1016/j. tcb.2015.05.002

32. Yu FX, Zhao B, Guan KL. Hippo pathway in organ size control, tissue homeostasis, and cancer. Cell. 2015;163(4):811-828. doi:10.1016/j. cell.2015.10.044 
33. Moya IM, Halder G. Hippo-YAP/TAZ signalling in organ regeneration and regenerative medicine. Nat Rev Mol Cell Biol. 2019;20(4):211-226. doi:10.1038/s41580-018-0086-yt

34. Zheng Y, Pan D. The hippo signaling pathway in development and disease. Dev Cell. 2019;50(3):264-282. doi:10.1016/j.devcel.2019.06.003

35. Kegelman CD, Mason DE, Dawahare JH, et al. Skeletal cell YAP and TAZ combinatorially promote bone development. FASEB J. 2018;32 (5):2706-2721. doi:10.1096/fj.201700872R

36. Hao J, Zhang Y, Wang Y, et al. Role of extracellular matrix and YAP/TAZ in cell fate determination. Cell Signal. 2014;26(2):186-191. doi:10.1016/ j.cellsig.2013.11.006

37. Tan FZ, Dai HL. TAZ accelerates osteogenesis differentiation of mesenchymal stem cells via targeting PI3K/Akt. Eur Rev Med Pharmacol Sci. 2019;23(3Suppl):81-88. doi:10.26355/eurrev_201908_18633

38. Park JS, Kim M, Song NJ, et al. A reciprocal role of the Smad4-Taz axis in osteogenesis and adipogenesis of mesenchymal stem cells. Stem Cells. 2019;37(3):368-381. doi:10.1002/stem.2949

39. Yang T, Zhang J, Cao Y, et al. Wnt5a/Ror2 mediates temporomandibular joint subchondral bone remodeling. J Dent Res. $2015 ; 94(6)$ :803-812. doi:10.1177/0022034515576051

40. Oishi I, Suzuki H, Onishi N, et al. The receptor tyrosine kinase Ror2 is involved in non-canonical Wnt5a/JNK signalling pathway. Genes Cells. 2003;8(7):645-654. doi:10.1046/j.1365-2443.2003.00662.x

41. Kikuchi A, Yamamoto H, Sato A, Matsumoto S. Wnt5a: its signalling, functions and implication in diseases. Acta Physiol (Oxf). 2012;204(1):1733. doi:10.1111/j.1748-1716.2011.02294.x

42. Person AD, Beiraghi S, Sieben CM, et al. WNT5A mutations in patients with autosomal dominant Robinow syndrome. Dev Dyn. 2010;239(1):327337. doi:10.1002/dvdy.22156

43. Afzal AR, Rajab A, Fenske CD, et al. Recessive Robinow syndrome, allelic to dominant brachydactyly type B, is caused by mutation of ROR2. Nat Genet. 2000;25(4):419-422. doi:10.1038/78107

44. Kikuchi A, Yamamoto H, Sato A. Selective activation mechanisms of Wnt signaling pathways. Trends Cell Biol. 2009;19(3):119-129. doi:10.1016/ j.tcb.2009.01.003

45. Mikels AJ, Nusse R, Arias AM. Purified Wnt5a protein activates or inhibits beta-catenin-TCF signaling depending on receptor context. PLoS Biol. 2006;4(4):e115. doi:10.1371/journal.pbio.0040115

46. Duan DY, Tang J, Tian HT, Shi YY, Jia J. Adipocyte-secreted microvesicle-derived miR-148a regulates adipogenic and osteogenic differentiation by targeting Wnt5a/Ror2 pathway. Life Sci. 2021;278:119548. doi:10.1016/j.lfs.2021.119548

47. Piccolo S, Dupont S, Cordenonsi M. The biology of YAP/TAZ: hippo signaling and beyond. Physiol Rev. 2014;94(4):1287-1312. doi:10.1152/ physrev.00005.2014

48. Wei Q, He M, Chen M, et al. Icariin stimulates osteogenic differentiation of rat bone marrow stromal stem cells by increasing TAZ expression. Biomed Pharmacother. 2017;91:581-589. doi:10.1016/j.biopha.2017.04.019

\section{Publish your work in this journal}

Drug Design, Development and Therapy is an international, peer-reviewed open-access journal that spans the spectrum of drug design and development through to clinical applications. Clinical outcomes, patient safety, and programs for the development and effective, safe, and sustained use of medicines are a feature of the journal, which has also been accepted for indexing on PubMed Central. The manuscript management system is completely online and includes a very quick and fair peer-review system, which is all easy to use. Visit http://www.dovepress.com/testimonials.php to read real quotes from published authors.

Submit your manuscript here: https://www.dovepress.com/drug-design-development-and-therapy-journal 